\title{
Reply to correspondence on: Laparoscopic resection rectopexy versus laparoscopic ventral rectopexy for complete rectal prolapse by H. A. Formijne Jonkers et al.
}

\author{
H. A. Formijne Jonkers • E. C. J. Consten • \\ S. D. Wexner
}

Received: 22 June 2014 / Accepted: 22 June 2014/Published online: 19 July 2014

(C) Springer-Verlag Italia Srl 2014

\section{Dear Sir,}

We would like to thank Prof. Bergamaschi and his associates for critically appraising our study.

Laparoscopic ventral rectopexy (LVR) and laparoscopic resection rectopexy (LRR) are probably currently the most common procedures performed for the surgical treatment of rectal prolapse (RP) in Europe and the USA, respectively [1]. Unfortunately, these techniques have not been compared in the literature.

We are well aware of the methodological limitations of our study, the retrospective nature foremost among them. Furthermore, because this study was undertaken in two different centers, there were many technical and care path differences, which could have influenced the results.

Strengths of the study include that comparison of outcomes was possible as functional results of both procedures for RP (incontinence and constipation/ODS) were meticulously documented in the charts of all patients, in both hospitals. In case of doubt, the Rome II criteria were used as a guide to determine whether a patient suffered from symptoms of constipation. Furthermore, complications were systematically registered in both centers, and discharge criteria were identical.

Realizing these limitations, our group has initiated the LaProS study. In this study, the results of LVR and LRR for RP are being prospectively compared in a large cohort of patients. Functional results are being qualified and quantified using validated questionnaires, both preoperatively and during follow-up.

While the smoke from the LaProS study will be much more powerful, the fumes from the "Laparoscopic resection rectopexy versus laparoscopic ventral rectopexy for complete rectal prolapse" study are quite enticing.

Conflict of interest None.

\section{Reference}

1. Formijne Jonkers HA, Draaisma WA, Wexner SD et al (2013) Evaluation and surgical treatment of rectal prolapse: an international survey. Colorectal Dis 15:115-119
H. A. Formijne Jonkers $(\bowtie) \cdot S$. D. Wexner

Department of Colorectal Surgery, Cleveland Clinic Florida,

Weston, FL, USA

e-mail: HA.Formijnejonkers@meandermc.nl

E. C. J. Consten

Department of General Surgery, Meander Medical Center,

Amersfoort, The Netherlands 